\title{
Digital videodensitometric measurement of aortic regurgitation
}

\author{
J. Divernois, P. Chatelain, P.-A. Doriot, B. Meier, W. Rutishauser \\ Cardiology Center. University Hospital, CH-1211 Geneva 4, Switzerland
}

KEY WORDS: Aortic regurgitation, videodensitometry, digital subtraction angiography, DSA.

A videodensitometric method for quantification of aortic regurgitation which requires neither measurement of cardiac output nor determination of enddiastolic and endsystolic left ventricular volumes has been developed. The injection of $20 \mathrm{ml}$ of contrast medium into the left ventricle is digitally recorded at 25 images $\mathrm{s}^{-1}$ during $20 \mathrm{~s}$ using an equipment for digital subtraction angiography (Digitron 2, Siemens). The Digitron computes 2 'time dilution curves' (TDC) from the unsubtracted image sequence, for 2 regions of interest drawn around the angiographic enddiastolic and endsystolic left ventricular silhouettes. Enddiastolic and endsystolic points of the $T D C$ are then entered into a VAX-750 computer, which calculates the ejection fraction $(E F)$, the forward ejection fraction (FEF) and the regurgitant fraction (RGF). This is performed by a complex fitting algorithm based on a physical model of the washout process of contrast medium, which reconstructs the two best enddiastolic and endsystolic baselines in the washout parts of the two TDC. The EF, FEF and RGF obtained in 9 regurgitant and 11 nonregurgitant patients have been compared with the corresponding values $E F_{\mathrm{v}} . F E F_{\mathrm{v}}$ and $R G F_{\mathrm{v}}$ obtained by a conventional technique (Cardiogreen and biplane $L V$ area-length volumetry). Regression analysis yielded: $E F=0.88 \times E F_{0}$ (regression line forced through the origin), $r=0.77, F E F=0.76 \times F E F_{v}+3, r=0.96$, $R G F=0.94 \times R G F_{v}+5, r=0.98$ (v stands for volumetry).

\section{Introduction}

Conventional quantification of aortic regurgitation requires determination of cardiac output $(\mathrm{CO})$ by an indicator dilution technique or by the Fick principle, and calculation of enddiastolic and endsystolic left ventricular volumes (EDV and ESV) from a ventriculogram. The regurgitant fraction (RGF) is obtained by comparing forward stroke volume $\mathrm{FSV}=\mathrm{CO} / \mathrm{HR}$ $(\mathrm{HR}=$ heart rate $)$ and stroke volume $\mathrm{SV}=\mathrm{EDV}-$ ESV according to the formula $\mathrm{RGF}(\%)=100 \times(\mathrm{SV}$. FSV)/SV.

This method has some disadvantages. For instance, the two required measurements are not performed simultaneously. Another limitation consists of the moderate accuracy of the calculated volumes EDV and ESV, inherent to the geometric model of the left ventricle (LV), and of the resulting poor accuracy of the stroke volume. This latter is additionally biased by the intra- and interobserver variability in the delineation of the angiographic enddiastolic and endsystolic silhouettes. The limited accuracy of routine cardiac output determination and conse-

Address for correspondence: Dr J. Divernois, Cardiology Center. Lniverssty Hospital, CH-1211 Geneva 4, Swizerland. quently of the forward stroke volume FSV represents a third limitation.

To overcome these drawbacks, other methods based on roentgenvideodensitometry have been proposed $^{[1-7.9]}$. Falliner and co-workers described a technique which requires neither $\mathrm{CO}$ measurement, nor LV volumetry ${ }^{[8]}$. They used an analog densitometer to integrate the logarithmic videotape-signal of the recorded angiographic sequence inside the outlined enddiastolic left-ventricular silhouette in each image, in order to obtain the 'densogram' or 'time dilution curve' (TDC) of the contrast medium injected into the LV. In particular, they described a solution to the problem of drifting baselines in the washout part of the TDC (explained below).

The method presented in this paper is similar to their technique. However, in order to improve the accuracy of the measured densitometric RGF, we tested several sophisticated solutions to the problem of drifting baselines. Our equipment for digital subtraction angiography (Digitron 2, Siemens) allows to define regions of interest (ROI) and to compute the corresponding TDC from the digitally recorded angiographic sequence. This simplifies apparative and operative aspects for roentgendensitometric volume and flow measurement. 


\section{Methods}

\section{THE DENSITOMETRIC APPROACH}

The basic principle of the densitometric method is to compute for each image of the angiographic sequence (selective $L V$ injection) a value $M(t)$ proportional to the amount of contrast medium contained in the LV at time t. The proportionality factor is irrelevant because subsequent operations involve only ratios of contrast medium amounts. The values $M(t)$ are obtained from a TDC as explained later. The TDC itself is obtained by summation of the digitized logarithmic video-signal $\log S(x, y)$ over all pixels $(x, y)$ of a ROI drawn around the enddiastolic silhouette of the LV, for each image of the sequence. Obviously constant radiographic settings of $\mathrm{KV}, \mathrm{mA}$ and videoamplification are a prerequisite. With some assumptions explicited below, the time dependent concentration of contrast medium in the left ventricular cavity, respectively the values $M(t)$ can be related to the corresponding LV volumes of interest (EDV and ESV), and to the regurgitant volume.

The assumptions are:

(1) The mitral valve is competent; (2) the concentration of contrast medium in the $\mathrm{LV}$ is homogeneous during each considered systole of the washout phase (the washout phase begins at the end of the injection); (3) the concentration of contrast medium in the regurgitant volume is equal to the concentration which was present in the LV during the systole which just termınated; (4) HR, T, (systole duration), EDV, ESV and RGF are constant during the part of the washout phase selected for determination of EF, FEF and RGF.

A schematic TDC aortic regurgitation is shown in the upper left panel of Fig. 1. The upward deflection of the curve, measured with respect to the baseline and beginning with onset of the injection, is the mentioned value $\mathbf{M}(\mathrm{t})$. Based on assumptions (1) to (4), one can show in this simplified case that the
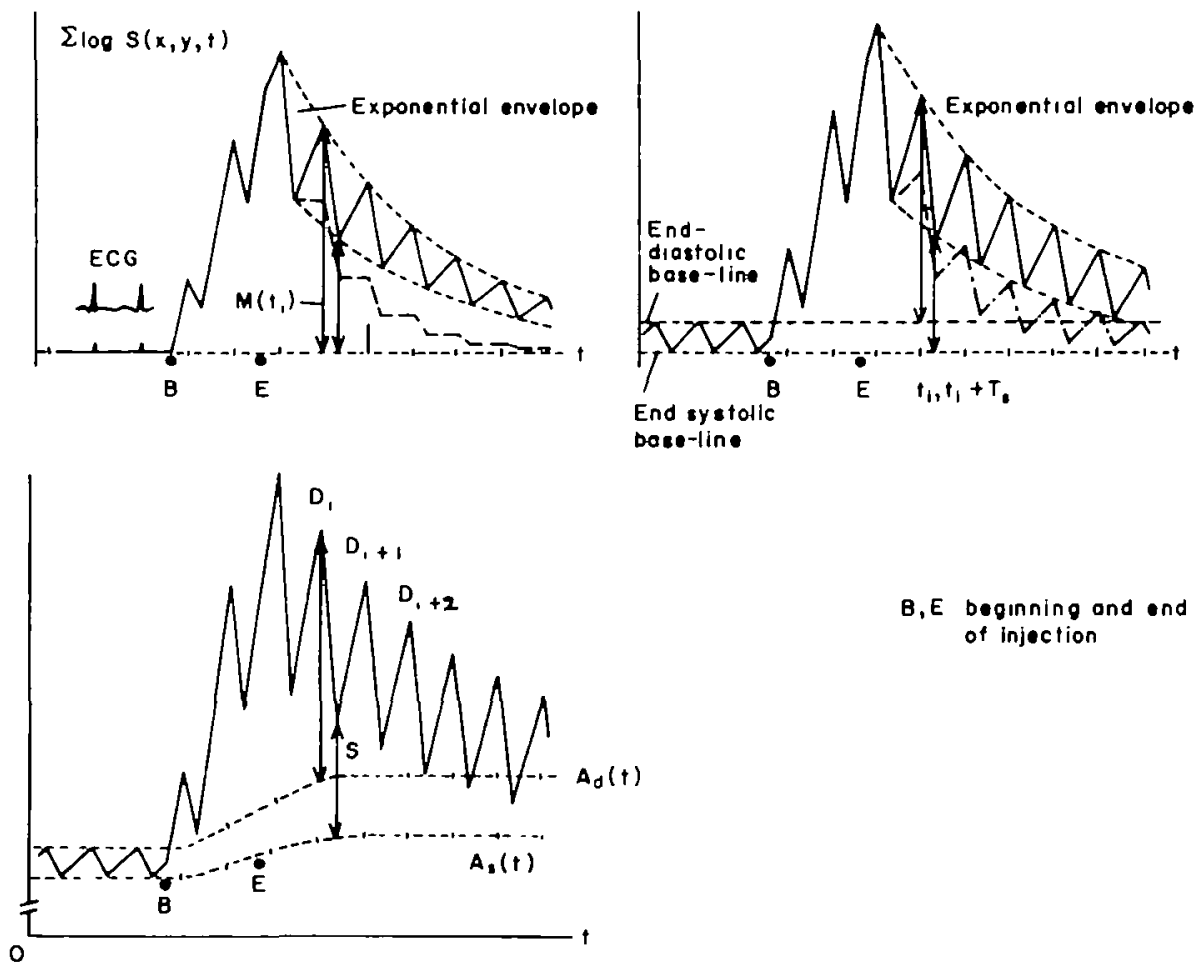

Figure I Schematic tıme dilution curves (TDC) in aortic regurgitation. Upper left panel: simplified TDC-the dotted line shows the constant baseline and the enddiastolic and endsystolic envelopes of exponential washout. The dashed line shows a hypothetical case without regurgitation. Upper rıght panel: The periodic oscallations of the density before contrast injection define enddiastolic and endsystolic baselines. Bottom panel: With differently drifting enddiastolic and endsystolic baselines. the exponential washout is calculated between the baselines and the non-exponential envelopes. (The ordinate is in arbitrary units ) 


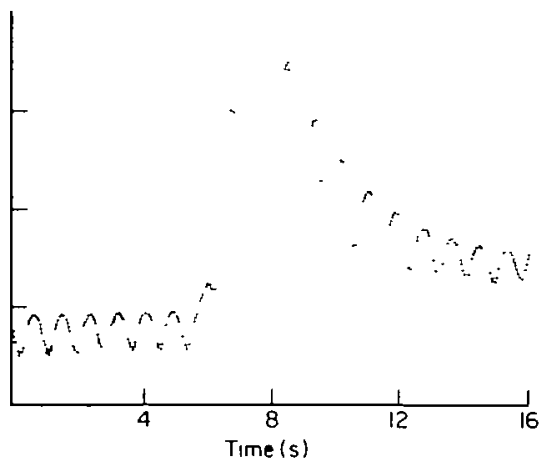

Figure 2 Real TDC of a female patient of 49 years with an aortic regurgitation fraction of $50 \%$, showing the considerable drift of the baseline (The ordinate is in arbitrary units).

enddiastolic (peaks) and endsystolic points (valleys) of the washout part lie on 2 exponentially decreasing curves (envelopes). The dashed line is the hypothetical TDC one would obtain if regurgitation disappeared at the end of the injection (the envelopes of this TDC are also exponentials). Unit and zero lines of the ordinate are arbitrary and have been skipped.

A minor problem consists in the fact that even in the absence of contrast medium in the $L V$, a real TDC presents a penodic oscillation, due to the varying absorption of involved tissues and ventricular blood during the cardiac cycle (see Fig. 2 and upper right panel of Fig. 1). Thus, the enddiastolic values $\mathbf{M}\left(\mathrm{t}_{\boldsymbol{i}}\right)$ and the endsystolic values $M\left(t_{l}+T_{s}\right)$ must be measured relatively to an enddiastolic and an endsystolic baseline (in dotted lines), respectively. The dashed line represents again the hypothetical TDC for disappearing regurgitation, of which the envelopes are still exponentials. A major problem consists in the drift of both baselines soon after onset of the injection, shifting the TDC progressively upwards (Fig. 2 and bottom panel of Fig. 1), with the result that enddiastolic and endsystolic points $\left(D_{i}\right.$ and $\left.S_{i}\right)$ of a real TDC do not lie on exponential envelopes. However, the previous 2 exponential envelopes can be found again in the equations

$M\left(t_{i}\right)=D_{i}-A_{d}\left(t_{i}\right)=B_{d} \exp \left[C_{i}\right]$,

$M\left(t_{i}+T_{s}\right)=S_{i}-A_{s}\left(t_{i}+T_{s}\right)=B_{s} \exp \left[C\left(t_{i}+T_{s}\right)\right]$

describing the envelopes of a real TDC. $A_{d}(t)$ and $A_{s}(t)$ are the (partially) unknown enddiastolic and endsystolic baselines defined relatively to the arbitrary zero line. If the unknown coefficients $B_{d}, B_{s}$ and $C$ of the 2 exponentials can be determined from the TDC, the ejection fraction (EF), the forward ejection fraction (FEF) and the regurgitant fraction (RGF) can be calculated according to the formulas:

$E F(\%)=\left[1-\left(B_{s} / B_{d}\right) \exp \left(C_{s}\right)\right] \times 100$

$\mathrm{FEF}(\%)=[1-\exp (\mathrm{CT})] \times 100$

( $\mathrm{T}$ is the period of the cardiac cycle in the washout phase)

$\operatorname{RGF}(\%)=[1-\mathrm{FEF} / \mathrm{EF}] \times 100$

Determination of $B_{d}, B_{s}$ and $C$ implies of course determination of $A_{d}(t)$ and $A_{r}(t)$ also.

A simpler approach would be to calculate EF, FEF and RGF from formulae involving only appropriate differences (Cf.[8]):

$$
\begin{aligned}
\mathrm{EF}_{i}(\%)=\left[1-\left(\mathrm{S}_{i}-\mathrm{S}_{i+1}\right) /\left(\mathrm{D}_{i}-\mathrm{D}_{i+1}\right)\right] \times 100 & =\mathrm{EF}(\%) \\
\mathrm{FEF}_{i}(\%)=\left[1-\left(\mathrm{D}_{i+1}-\mathrm{D}_{i+2}\right) /\left(\mathrm{D}_{i}-\mathrm{D}_{i+1}\right)\right] \times 100 & =\mathrm{FEF}(\%) \\
\mathrm{RGF}_{i}(\%)=\left(1-\mathrm{FEF}_{i} / \mathrm{EF}_{i}\right) \times 100 & =(1-\mathrm{FEF} / \mathrm{EF}) \\
& \times 100=\operatorname{RGF}(\%)
\end{aligned}
$$

Thus, 2 adjacent cardiac cycles would yield one set of values $E F, F E F$ and $R G F$, whereby the fiability of the obtained EF, FEF and RGF would decrease rapidly with increasing order number $i$ of the pair of cardiac cycles (Cf.[8]). An important underlying assumption of this solution is that the 2 unknown baseline portions corresponding to the considered pair are horizontal.

A major disadvantage is that all involved differences must be highly accurate, for simple mathematical reasons. Since this was not the case in our study, we first designed a computer fitting algorithm to find 2 horizontal baselines portions for the washout part of the TDC, such that the $D_{i}$ and $S_{i}$ would lie best on the curves

$$
\begin{aligned}
& D_{i}=A_{d}+B_{d} \exp \left[C_{i}\right] \text { and } \\
& \qquad S_{l}=A_{s}+B_{s} \exp \left[C\left(t_{i}+T_{s}\right)\right],
\end{aligned}
$$

where $A_{d}(t)=A_{d}=$ constant and $A_{r}(t)=A_{s}=$ constant. This allowed to operate globally with all available cardiac cycles and to obtain the coefficients $B_{d}, B_{s}$ and $C$ needed to calculate the EF, FEF and RGF according to equations (2), (3) and (4). However, this approach sometimes led to irrealistic baselines, suggesting that the drifted true baselines were not simply horizontal as assumed. It was therefore abandoned.

The following method was finally judged appropriate. The principle of the algorithm is also to find 2 baselines such that the enddiastolic and endsystolic 


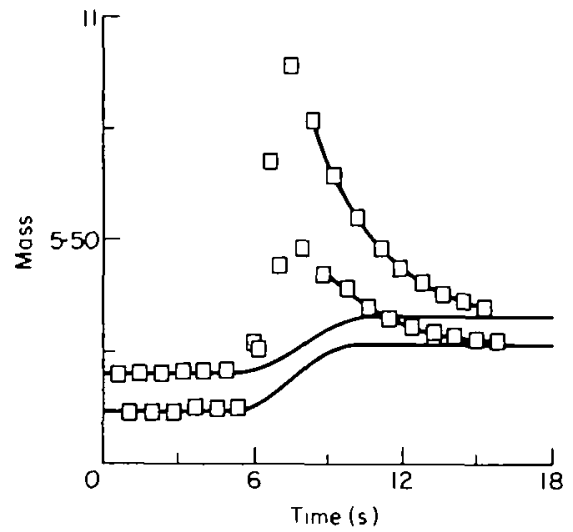

Figure 3 Reconstruction of the 2 drifted baselınes by the fitting algorithm, for the patient shown in Fig. 2

points $D_{i}$ and $S_{i}$ measured relatively to these new baselines lie best on the exponential curves described by the equation pair (1). However, each baseline is now composed of a sinusordal segment starting at the end of the true baseline segment (begin of the injection) and of a straight segment (Fig. 3). The beginning of the sinusoidal part is not critical and is set by the operator at the approximate start of the injection. The end of the straight segment can be chosen by the operator, or set by the program at the lowest endsystolic point of the washout part. The best amplitude and best wavelength of each sinusoid, as well as the best coefficients $B_{d}, B_{s}$ and $C$, are found by the fitting algorithm using the criterion above. The two resulting exponential curves correspond to the equation pair (1), and EF, FEF and RGF are now calculated using formulas (2), (3) and (4).
The various algorithms developed to reconstruct the unknown baselines were tested on many simulated TDC similar to the one of Fig. I, bottom panel. With the retained algorithm, the obtained values EF, FEF and RGF were always within a few percents of the expected ones Variations of the results secondary to simulated irregularities such as extrasystoles, unstable cardiac period etc. were observed. However, they were within acceptable limits.

\section{APPLICATION TO PATIENTS}

10 consecutive patients with clinical aortic regurg1tation and free of mitral regurgitation were examıned. 18 patients without suspected aortic or mitral regurgitation (called 'normals' in the following) were also investigated, to assess the performances of the method for very small or zero regurgitation, which cannot be measured accurately by the conventional and by densitometric techniques with left ventricular injection but by roentgendensitometric methods using aortic injection $^{[1-9]}$. The first patients (regurgitants and normals) received intraventricular injections of $40 \mathrm{ml}$ of Iohexol (Omnipaque- $350,15 \mathrm{ml} \mathrm{s}^{-1}$ ). We lowered then the dose to $20 \mathrm{ml}\left(10 \mathrm{ml} \mathrm{s}^{-1}\right)$ to reduce non-linear densitometric effects. The sequence was recorded on the Digitron in continuous mode, which provides constant radiographic parameters $(\mathrm{kV}, \mathrm{mA}$, video amplification) durng the sequence. Imaging rate was 25 frames s $^{-1}$ and resolution $256 \times 256 \times 10$ bits. The injection was delayed by $5 \mathrm{~s}$ to secure the needed 2 initial baselines (2 $\mathrm{s}$ are needed to regulate the exposure parameters). The sequence duration was $20 \mathrm{~s}$.

In prelıminary investigations sometimes artıficial aortic regurgitation induced by the catheter was found. Therefore the catheter was pulled back into
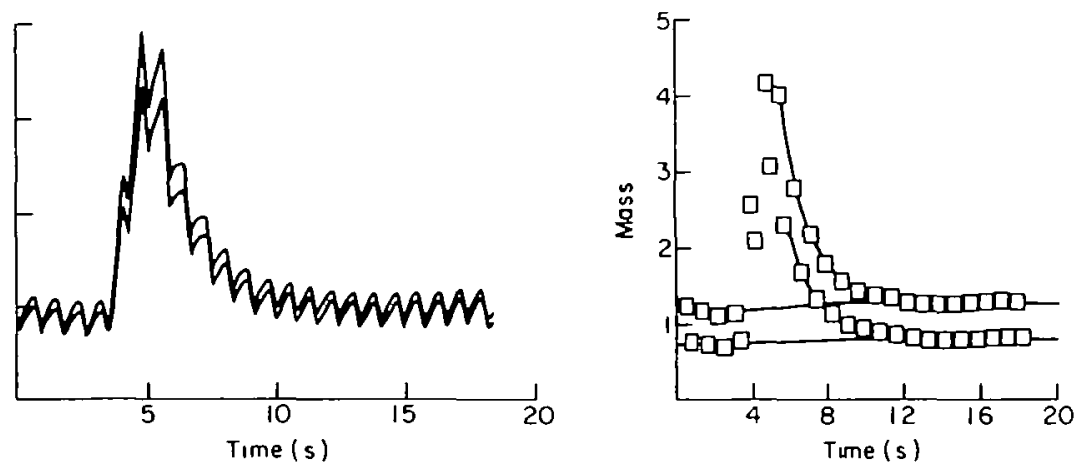

Figure 4 Left panel: enddiastolic and endsystolic TDC from a patıent without aortic regurgitatıon. right panel: computer reconstruction of this case. 
the aorta at the end of the power injection in all normal patients. Each patient also had biplane LV cineangiography (RAO $30^{\circ}$ and $\mathrm{LAO} 60^{\circ}$ projections), $15 \mathrm{~min}$ before or after the Digitron sequence. Cardiac output was measured in all patients with regurgitation from two Cardiogreen injections into the left ventricle with sampling in the femoral artery.

Using the available Digitron software, 2 ROIs were drawn around the enddiastolic and endsystolic silhouettes of the LV on adequate mask subtracted images, and the corresponding 2 TDC were computed from the original (unsubtracted) image sequence (Fig. 4). Introduction of an endsystolic ROI allows for elimination of the aortic valve downward movement during systole. (The incursion of the first part of the ascending aorta into the enddiastolic ROI during systole produces an artificial regurgitation if only an enddiastolic window is used).

The 2 computed TDC being automatically normalized to the pixel area by the supplied Digitron software, the TDC do not yield the points $D_{i}$, resp. $S_{l}$, but the points $D_{t, \text { aorm }}$, resp. $S_{l \text {,arm }}$. The points $D_{l, \text { norm }}$ measured on the enddiastolic TDC (see Fig. 4), and the points $S_{i, \text { norm }}$ measured on the endsystolic TDC were entered from the multispot film document (Digitron) by means of a graphic tablet into a VAX-750 computer. The enddiastolic and endsystolic points of the inttial baselines were also digitized. The surfaces of the 2 ROIs, also registered number of pixels), were used to 'denormalize' the points $D_{i, \text { norm }}$, resp. $S_{i, \text { norm }}$, in order to obtain the points $D_{i}$, resp. $S_{i}$. The obtained points $D_{i}$ and $S_{i}$, and the denormalized points of the initial baselines are used by the complex algorithm for determination of the 2 best baselines $A_{f}(t)$ and $A_{s}(t)$, and simultaneously of the best coefficients $B_{d}, B_{s}$ and $C$, as previously explained. The (vertical) distances of these points relatively to the baselines found are the $M\left(t_{i}\right)$ and $M\left(t_{i}+T_{s}\right)$ of equation pair (1). The EF, FEF and RGF are finally calculated according to equations (2), (3) and (4).

The $\mathrm{EDV}_{v}, \mathrm{ESV}_{\mathrm{v}}$, and $\mathrm{EF}_{\mathrm{v}}$ were obtained in the conventional way using the area-length method ( $v$ stands for 'volumetry'). In 3 patients with inadequate LAO $60^{\circ}$ view, calculations were performed using the RAO $30^{\circ}$ view only. Determination of CO from the Cardiogreen dilution curves was performed using the standard formula, and $\mathrm{RGF}_{\nu}$ was calculated according to the formula given in the introduction. All
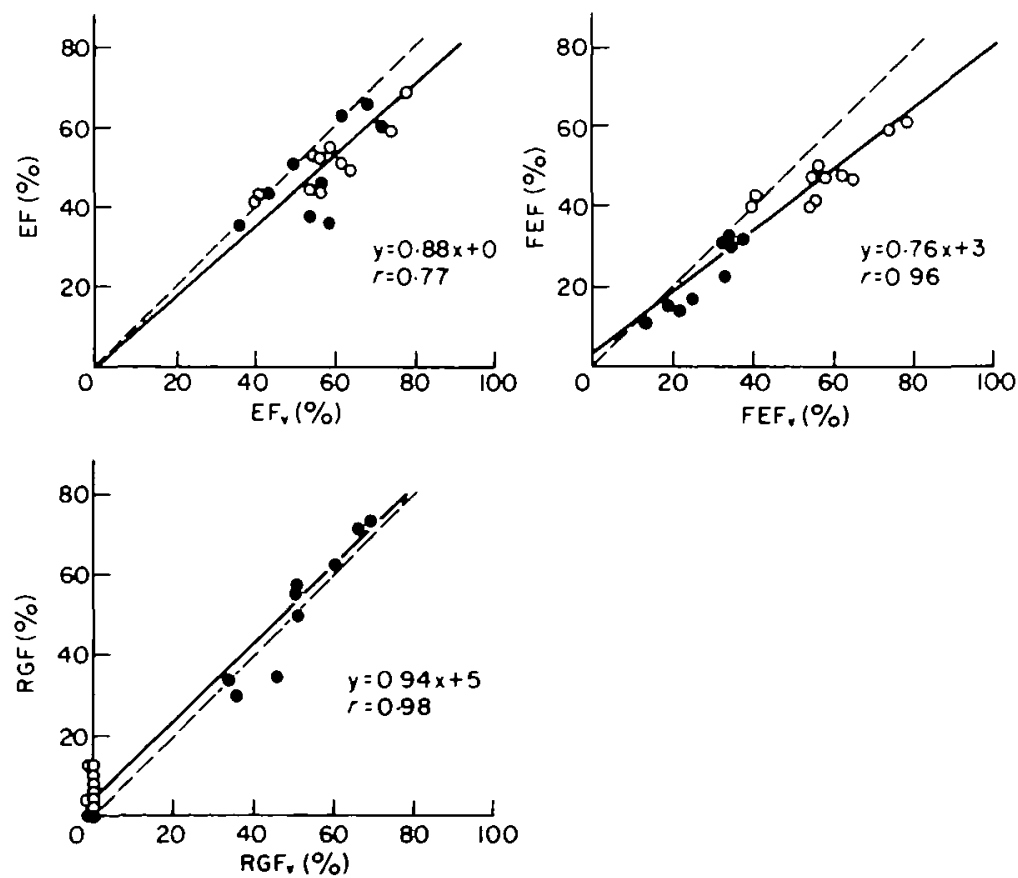

Figure 5 Correlation between ejection fractions obtained by videodensitometry (EF) and conventional method $\left(E F_{v}\right)$. Upper right panel-correlation between the forward ejection fractions FEF and $F E F_{p}$; bottom panel-the same for the regurgitation fractions RGF and $R G F_{p}$. 


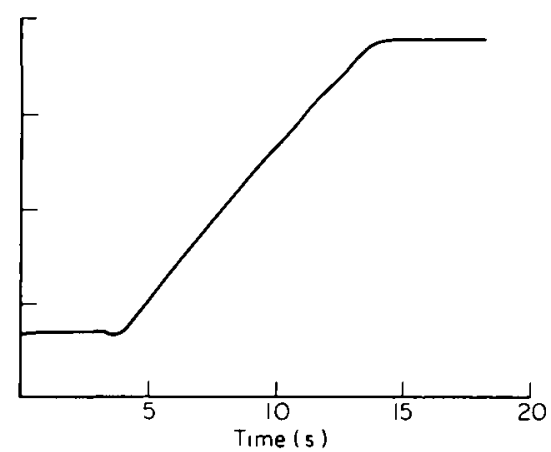

Figure 6 TDC obtained by inflatung a balloon by a $1 \cdot 10$ diluted Omnipaque 350 solution from 0 to $200 \mathrm{ml}\left(20 \mathrm{ml} \mathrm{s}^{-1}\right)$. No appreciable non-linearity is observed.

conventional data have been calculated by cardiologists of our catheterisation laboratory, in the usual fashion and independently. They were gathered from the patient reports for comparison with our method, which was thus truly compared with routine clinical results. From the initial 28 patients, 8 were excluded because of irregular cardiac rhythm in the washout phase (atrial fibrillation, supraventricular or ventricular extrasystoles), respiration movements displacing the LV silhouettes out of the ROI's, or for other reasons mentioned below. Finally, 9 regurgitant and 11 normal patients were retained.

\section{Results}

A plot of the obtained densitometric ejection fractions $E F$ versus the conventional $E F_{v}$ is shown in the upper left panel of Fig. 5. The regression line (in plain line) has been forced through the origin because of the small range of the EFv values. The correlation between densitometric forward ejection fractions FEF and conventional $\mathrm{FEF}_{\boldsymbol{v}}$ is given in the upper right panel. The bottom panel shows the regurgitant fraction $R G F$ versus the $R G_{p}$.

\section{Discussion}

The densitometric EF are lower than the $\mathrm{EF}_{\mathrm{v}}$ obtained by biplane or monoplane volumetry (arealength method). An immediate explanation would be that the $\mathrm{M}(\mathrm{t})$ are not proportional to the true amounts of contrast medium. However, inflating a balloon placed in a water bath $(20 \mathrm{~cm}$ depth) with a $1: 10$ diluted contrast medium solution, from $0 \mathrm{ml}$ to $200 \mathrm{ml}\left(20 \mathrm{ml} \mathrm{s}^{-1}\right)$, under the same radiographic conditions as during the recording from patients, yielded an almost perfectly straight line as shown in Fig. 6. So, there is no evidence for non-linearity with these settings in our system. Another reason would be that assumption (3) is not fulfilled, but this may produce lower FEF but not lower EF. Thus, we offer 3 hypothetical explanations:

(a) Some contrast medium remains trapped in the LV cavity during the washout process, so that the stroke volume would not be recruited in proportion to the contrast medium. This could be due to undyed blood coming from the left atrium around the anterior mitral leaflet which is preferably ejected during systole. Those effects, however, should be minimized by the additional mixing due to the aortic regurgitation.

(b) Concomitant opacification of the myocardium occurs (but the reconstruction of the base lines takes care of this effect).

(c) The EF are accurate and the $E F_{0}$ overestimate the true ejection fractions. It is of interest that the results of Falliner and co-workers ${ }^{[8]}$ were quite similar to ours as far as trends are concerned.

The densitometric RGF are in good agreement with the $\mathrm{RGF}_{v}$.

The FEF are lower than the $\mathrm{FEF}_{\mathrm{p}}$. If the EF really underestimates the true EF by correct RGF, then the FEF also underestimates the true FEF. Underestimation of the true FEF is also possible by correct EF if assumption (3) is not well fulfilled (if the concentration of contrast medium in the regurgitant volume would be higher than in the just previously ejected volume). But this would imply that the RGF are too high. Moreover, the obtained FEF are accurate if one accepts the EF and RGF results.

More generally, acceptance of any two of the 3 sets of results EF, FEF and RGF implies acceptance of the third also, since the RGF were obtained from the $E F$ and FEF [equation (4)].

In the introduction, some unsatisfying aspects of the conventional method were pointed out. However, an advantage of this latter over the densitometric approach is its greater tolerance for irregularities of the cardiac cycle. Marked irregularities in the TDC produced EF, FEF and RGF values which were clearly different from the expected values $E F_{\mathfrak{v}}, F F_{0}$ and $R F_{v}$ (these patients were therefore excluded). Some erroneous values could also be attributed to respiratory movements during the Digitron sequence, confirmed by retrospective examination of the sequence recorded also on videotape (these patients were also excluded).

Inversely, 2 cases of regurgitation obtained from 
correct TDC by presumed normal patients were plausible: The first patient had undergone an aortic commissurotomy. The other patient had presented massive mitral regurgitation on a cineangiogram performed 6 months prior to the study, after an infarct of the inferior wall. However, he had no slight regurgitation during the conventional ventriculography. Both were excluded from the study.

\section{Conclusion}

The method described in this paper shows an excellent correlation between densitometrically and conventionally measured aortic regurgitation fractions. The main reason of this lies in the performed reconstruction of the drifting baselines and in the introduction of an endsystolic ROI. The ejection fractions and forward ejection fractions are also well correlated with the corresponding conventional values, but in average lower. Explanations are offered but densitometry is probably more accurate than conventional methods.

\section{References}

[1] Rutishauser W. Aortenınsuffizenz. In. Kreislaufanalyse mittels Roentgendensitometrie Hans Huber Verlag. $1969 ; 89-92$.

[2] Von Bernuth G, Tsakırs AG, Wood EH. Quantitation of experimental aortic regurgitation by videodenst- tometry in intact dogs. Circulation 1970: $41 / 42$ (Suppl III) 60 .

[3] Bürsch JH, Heıntzen PH. Simon R. Roentgenvideodensitometric determination of the residual fraction of the normal left ventncle and regurgitation fraction in expenmental aortic regurgitation. Physiologist 1972; 15: 3.

[4] Simon R, Steiger U, Wirz P, Krayenbūhl HP, Schōnbeck $M$, Rutishauser W. Quantifizierung von Aortenklappenınsuffizienzen durch videodensitometrische Kontrastmittelmengenmessungen. Schweiz Med Wochenschr 1974; 104: 1562-64.

[5] Bürsch JH. Heintzen PH. Some principles for circulatory studies using videodensitometry. In: Heintzen PH, Bürsch JH, eds. Roentgen-video-techniques for dynamic studies of structure and function of the heart and circulation, 2nd international conference. Stuttgart: Georg Thieme, 1978; 2-11.

[6] Wessel A, Faltz Ch, Bürsch JH. Videodensitometric and videometric studies of regurgitation and left ventricular volume in experimental aortic insufficiency. In: Heintzen $\mathrm{PH}$, Bürsch JH, eds. Roentgen-video-techniques for dynamic studies of structure and function of the heart and circulation, 2nd international conference. Stuttgart: Georg Thieme, 1978; 80-4.

[7] Simon R. Khnische Kontrastmittel-Densitometrie. Habilitationsschrift. Medizinische Hochschule Hannover $1981 ; 61-3$.

[8] Falliner A, Bürsch JH, Wessel A, Faltz HC, Heintzen PH Zuverlässigkeit der Roentgen-videodensitometrie für Klappeninsuffizienzmessungen und für die Bestimmung der ventrkulären Auswurffraktıon. Z Kardiol 1981; 70: 754-60.

[9] Erikson U. Densitometry in the diagnosis of aortic incompetence. Acta Radiol Diagn 1976; 17: 361-8 